\title{
Electrochemical Oxidation of Ethanol at Nickel Hydroxide Electrodes in Alkaline Media Studied by Electrochemical Impedance Spectroscopy
}

\author{
Jae-Woo Kim ${ }^{\#}$ and Su-Moon Park* ${ }^{\dagger}$ \\ Department of Chemistry and Center for Integrated Molecular Systems, \\ Pohang University of Science and Technology, Pohang 790-784, Korea
}

(Received June 2, 2005 : Accepted July 6, 2005)

\begin{abstract}
Electrochemical oxidation of ethanol at nickel electrodes has been studied in $1 \mathrm{M} \mathrm{KOH}$ solution containing $0.20 \mathrm{M}$ ethanol using electrochemical impedance spectroscopy. Equivalent circuits have been worked out by simulating the impedance data, and the results were used to model the oxidation of ethanol as well as the passivation of the electrode. The maximum rate of oxidation of $\mathrm{Ni}(\mathrm{OH})_{2}$ to $\mathrm{NiOOH}$ was observed at about $0.37 \mathrm{~V}$ vs. $\mathrm{Ag} / \mathrm{AgCl}$ reference electrode, while the maximum rate of ethanol oxidation at the $\mathrm{Ni}$ electrode was observed at about $0.42 \mathrm{~V}$. The charge-transfer resistance for oxidation of the electrode itself became smaller in the presence of ethanol than in its absence. These results suggest that the $\beta-\mathrm{Ni}(\mathrm{OH})_{2} / \beta-\mathrm{NiOOH}$ redox couple is acting as an effective electron transfer mediator for ethanol oxidation. The kinetic parameters also were obtained by the experimental and simulated results.
\end{abstract}

Keywords: Ethanol oxidatio, Nickel Hydroxide Electrode, Impedance

\section{Introduction}

Ethanol is oxidized to acetic acid via acetaldehyde at nickel electrodes. ${ }^{1}$ Most of the acetaldehyde is oxidized to acetic acid because the rate of oxidation of acetaldehyde is faster than that of ethanol. The oxidation reaction of organic compounds with oxides of higher valences is usually the rate-determining step. A redox couple of nickel, i.e., nickel hydroxide $\left(\mathrm{Ni}(\mathrm{OH})_{2}\right)$ and nickel oxyhydroxide $(\mathrm{NiOOH})$, was shown to be involved in the oxidation of alcohol at nickel electrodes in alkaline media. ${ }^{1}$ Anodic oxidation of nickel in alkaline media has been studied extensively for a better understanding of the passivation phenomena and charge/ discharge mechanism of nickel electrodes in batteries using various techniques such as the cyclic voltammetry, ${ }^{2-6}$ in-situ spectroelectrochemical methods, ${ }^{7,8}$ X-ray studies, ${ }^{9-13}$ and electrochemical impedance spectroscopy (EIS). ${ }^{14-25}$ Nickel is covered with a hydroxide/oxyhydroxide layer which is hydrated to a varying degree and whose stoichiometry and oxidation states depend on electrode potential and history. ${ }^{2-4,7,8}$ At a potential close to the hydrogen equilibrium potential, highly hydrated nickel hydroxide, $\alpha-\mathrm{Ni}(\mathrm{OH})_{2}$, in which water molecules form a disordered interlamellar layer, is formed. This compound transforms both with time and with increasing potentials to a less hydrated and more stable phase, $\beta$ -

\footnotetext{
" Present address: Science and Technology Cooperation, Hampton, Virginia 23666

*The Korean Electrochemical Society Active Member.
}

†E-mail: smpark@postech.edu
$\mathrm{Ni}(\mathrm{OH})_{2}$. The basic electrochemical reaction at the nickel electrode involves the oxidation of $\beta$-nickel hydroxide $\left(\mathrm{Ni}(\mathrm{OH})_{2}\right)$ to $\beta$-nickel oxyhydroxide $(\mathrm{NiOOH})$ on the passive film, but the chemistry can be further complicated by higher oxidation states, oxygen evolution, and the presence of different structural forms. The equivalent circuits simulated from the experimental impedance data were very complicated due to the complexity of the reaction.

The anodic oxidation of nickel in alkaline solutions has been modeled as porous electrodes in past studies with EIS. . $^{15,17,18,26-28}$ Various mathematical models were proposed for impedance responses of porous nickel electrodes. Chao et al. proposed the point defect model based on the movement of point defects in an electrostatic field for the growth behavior of a passive film on a metal surface. ${ }^{26-28}$ They demonstrated that the reaction at the film/solution interface is predominant at high frequencies and, thus, a variety of semicircles can be observed in the complex impedance plane. At low frequencies, the transport of point defects in the passive film is rate contro1ling, and a Warburg-type impedance spectrum is predicted on a passive film of $\mathrm{Ni}$ in aqueous buffer systems. Lenhart et al. reported the transmission line model for semi-infinite cylindrical pores using the average of pore electrolytes and solid phase resistances. ${ }^{15}$ In addition, Macdonald et al. suggested a nonuniform transmission line model that represents a single pore in a conical porous electrode by a nonuniform transmission line involving both nickel hydroxide and oxyhydroxide phases. ${ }^{17,18}$ The existing models do not completely satisfy the experimental data due to the assumptions made while setting up the models. 
In our previous work, we found that the $\mathrm{RuO}_{2}$-modified $\mathrm{Ni}$ electrode was efficient for the oxidation of ethanol, and that nickel facilitated the electrooxidation of ethanol at thermally prepared $\mathrm{RuO}_{2}$-modified $\mathrm{Ni}$ electrodes in alkaline media. ${ }^{29}$ Therefore, the impedance responses of the electrooxidation of ethanol as well as the passive films formed on the nickel electrode are important in understanding the mechanisms of a direct ethanol fuel cell (DEFC). In this work, we offer a detailed examination of the impedance responses of nickel hydroxide/ oxides reaction and electrooxidation of ethanol as a function of frequency and potential. The reactions of ethanol oxidation as well as the passivation of electrodes were modeled using equivalent circuits, and interpretations were made with the parameters used to fit the observed EIS behaviors.

\section{Experimental}

A nickel wire (Johnson Matthey, 99.99\%) with a diameter of $1.0 \mathrm{~mm}$ was used as a working electrode. Preparation of the nickel electrode was similar to that described in our previous work. ${ }^{29}$ The working electrode was first polished with $3 \mu \mathrm{m}$ alumina powder (Fisher Scientific), successively down to $1 \mu \mathrm{m}$, cleaned with ethanol, degreased with acetone, and finally washed with doubly distilled water in an ultrasonic bath (Cole Parmer 18001) for $30 \mathrm{~min}$. The wire was partially sealed with a heat-shrinkable Teflon tubing, exposing only the active site. Doubly distilled water and reagent grade chemicals were used for the preparation of the solutions. Each solution was purged with nitrogen for $10 \mathrm{~min}$ before measurements.

Cyclic voltammetric experiments were carried out in a $1 \mathrm{M}$ $\mathrm{KOH}$ solution containing $0.20 \mathrm{M}$ ethanol at room temperature. The cyclic voltammograms (CVs) were recorded using an EG\&G Princeton Applied Research (PAR) model 283 potentiostat/galvanostat, which was controlled by PAR $270 \mathrm{M}$ electrochemistry software. The working electrode was prepared according to the above procedure and an $\mathrm{Ag} / \mathrm{AgCl}$ (in saturated $\mathrm{KCl}$ ) electrode was used as a reference electrode. A platinum spiral wire was used as a counter electrode. The reference electrode was separated from the $\mathrm{KOH}$ electrolyte solution by an incomplete seal through a platinum wire in a Pyrex glass tubing and was located close to the working electrode in the current path between working and counter electrodes.

The EIS experiments employed an EG\&G model 283 potentiostat/galvanostat in conjunction with a Solartron SI 1255 HF Frequency Response Analyzer, which was driven by EG\&G M398 electrochemical impedance software. The ac amplitude was $5 \mathrm{mV}$ for all frequency ranges. The measurements were made at a rate of ten points per decade between $100 \mathrm{kHz}$ and $0.01 \mathrm{~Hz}$ at a stationary electrode. Impedance spectra were measured in $1 \mathrm{M} \mathrm{KOH}$ after steady-state CVs were obtained.

For an analysis of impedance data, a software program, "Equivalent Circuit," provided through EG\&G by University of Twente ${ }^{30}$ was used. The program simulated a variety of electrical circuits to numerically fit the measured impedance data. The program, combined with the general nonlinear least squares fitting procedure, allowed us to construct equivalent circuits, whose simulated responses fit the measured data well. Details of circuit analysis using this program and their applications to the interpretation of electrode/electrolyte interfaces have been described elsewhere. ${ }^{31-34}$

\section{Results}

Impedance measurements for oxidation of nickel-A steadystate $\mathrm{CV}$ acquired at a nickel electrode in $1 \mathrm{M} \mathrm{KOH}$ is shown in Fig. 1, which is in good agreement with those published in the literature. ${ }^{3-8}$ The anodic peak appeared at $0.40 \mathrm{~V}$ vs. $\mathrm{Ag} / \mathrm{AgCl}$ and the cathodic peak at $0.27 \mathrm{~V}$.

The electrochemical impedance spectra for the oxidation of nickel hydroxide to nickel oxyhydroxide in $1 \mathrm{M} \mathrm{KOH}$ were recorded between 0.24 and $0.47 \mathrm{~V}$ vs. $\mathrm{Ag} / \mathrm{AgCl}$ at potentials marked on the CV shown in Fig. 1. The potential was stepped anodically from $0.24 \mathrm{~V}$ to a point of interest and then an impedance spectrum was recorded. By graphical analysis and a complex nonlinear least squares (CNLS) fitting procedure, the equivalent circuits were derived from the simu-lation of the experimental data. All the parameters obtained from the simulation are listed in Table I.

The complex impedance spectra recorded at 0.24 and $0.28 \mathrm{~V}$ are shown in Fig. 2. The $\mathrm{R}_{\mathrm{s}}$ component, which is $3.8 \pm 0.2 \Omega$ is the solution resistance obtained from the high frequency intercept on the real reactance axis. ${ }^{34}$ The $n$ value was 1.0 or close to 1.0 for constant phase elements (CPEs), $Q_{1}$ and $Q_{4}$, both of which must be double layer capacitors. The equivalent circuit shown in Fig. $2 b$ represents a faradaic reaction taking place at these potentials with a polarization resistance of $R_{B}$ which is $R_{1}$ in the equivalent circuit shown. We believe that $\mathrm{Q}_{2}$ is a double-layer capacitor developed in the inner pores in a frequency range not affected by the porosity of the passive film, while $Q_{1}$ is a double layer capacitor developed in the outer pores of the film. A Warburg component with an $n$ value close to 0.5 (the element with a symbol $\mathrm{Q}_{3}$ in the circuits) is observed in most of the potential ranges

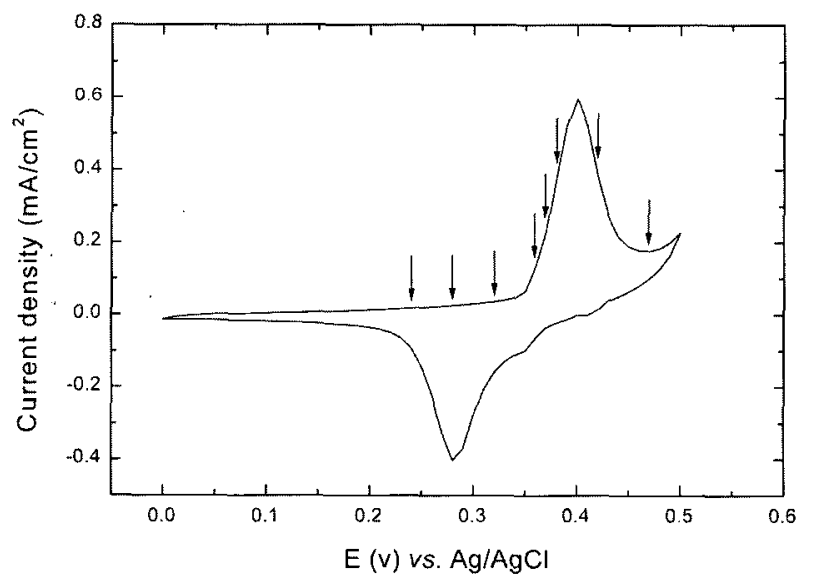

Fig. 1. Cyclic voltammogram of a nickel electrode $\left(\right.$ area $\left.=0.15 \mathrm{~cm}^{2}\right)$ in $1 \mathrm{M} \mathrm{KOH}$ recorded at $10 \mathrm{mV} / \mathrm{s}$. Impedance measurements were made at potentials marked. 
Table I. Parameters of Equivalent Circuits for Simulation of the Elements in $1 \mathrm{M} \mathrm{KOH}$.

\begin{tabular}{cccccccccccccccc}
\hline & & \multicolumn{2}{c}{$\mathrm{Q}_{1}$} & \multicolumn{2}{c}{$\mathrm{Q}_{2}$} & \multicolumn{2}{c}{$\mathrm{Q}_{3}$} & \multicolumn{2}{c}{$\mathrm{Q}_{4}$} & \multicolumn{2}{c}{$\mathrm{Q}_{5}$} \\
\hline $\mathrm{E}_{\text {applied }}(\mathrm{V})$ & $\mathrm{R}_{\mathrm{P}}(\Omega)^{\mathrm{b}}$ & $\mathrm{R}_{2}(\Omega)$ & $\mathrm{Y}_{\mathrm{Q}} \times 10^{-5}$ & $n$ & $\mathrm{Y}_{\mathrm{Q}} \times 10^{-5}$ & $n$ & $\mathrm{Y}_{\mathrm{Q}} \times 10^{-4}$ & $n$ & $\mathrm{Y}_{\mathrm{Q}} \times 10^{-4}$ & $n$ & $\mathrm{Y}_{\mathrm{Q}} \times 10^{-5}$ & $n$ & $\tau(\mathrm{s})$ & $\chi^{2}\left(\times 10^{-4}\right)^{\mathrm{c}}$ \\
\hline 0.24 & 2233.6 & - & 1.07 & 1.00 & 22.92 & 0.85 & 21.43 & 0.49 & 3.29 & 1.00 & 8.05 & 0.48 & 0.619 & 13.9 \\
0.28 & 2016.7 & - & 3.87 & 0.92 & 24.07 & 0.81 & 59.62 & 0.36 & 48.81 & 0.86 & 0.14 & -0.14 & 0.590 & 4.1 \\
0.32 & 1458.4 & - & 28.58 & 0.79 & 6.49 & 1.00 & 16.74 & 0.39 & 6.39 & 0.73 & - & - & 0.400 & 1.5 \\
0.36 & 1055.9 & - & 19.09 & 0.84 & 31.32 & 0.84 & 182.28 & 1.00 & 7.15 & 0.67 & - & - & 0.326 & 63.2 \\
0.37 & 660.2 & - & 10.22 & 0.81 & 66.67 & 0.85 & 6.24 & 0.60 & 954.65 & 0.71 & - & - & 0.269 & 2.9 \\
0.38 & 1666.3 & - & 66.03 & 0.74 & 1.54 & 0.67 & 14.50 & 0.48 & 19.07 & 0.65 & - & - & 0.425 & 4.1 \\
0.42 & 1811.6 & - & 64.61 & 0.77 & 37.01 & 1.00 & 8.11 & 0.54 & 2226.90 & 0.54 & - & - & 2.779 & 9.7 \\
0.47 & 1437.9 & 5817.1 & 1.48 & -1.00 & 137.17 & 0.81 & 146.61 & 1.00 & 34.29 & 0.67 & - & - & 4.422 & 5.4 \\
\hline
\end{tabular}

${ }^{\mathrm{a}} \mathrm{R}_{\mathrm{S}}$ values are not listed as they are almost constant at $3.8 \pm 0.2 \Omega$ for measurements made between 0.24 and $0.47 \mathrm{~V}$.

${ }^{b} R_{P}$ is $R_{1}$ at all applied potentials.

${ }^{c}$ Chi square values for the equivalent circuits fitting.

Electrode area was $0.15 \mathrm{~cm}^{2}$.

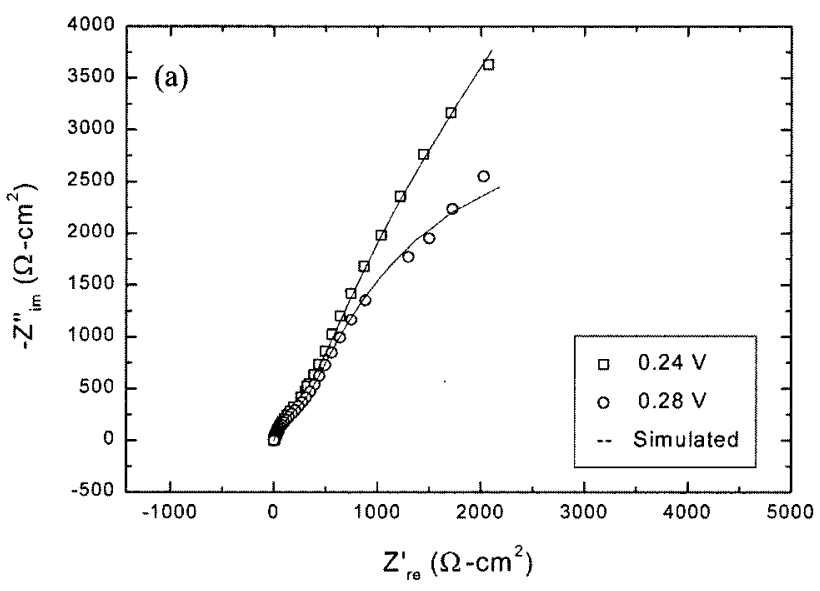

(b)

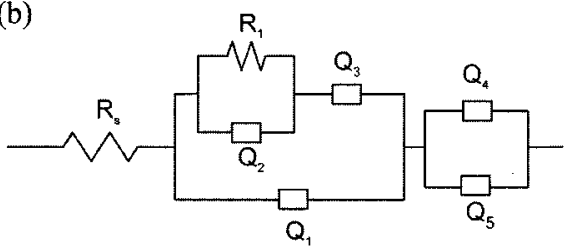

Fig. 2. (a) Complex impedance spectrum for oxidation of the nickel electrode at 0.24 and $0.28 \mathrm{~V}$ in $1 \mathrm{M} \mathrm{KOH.} \mathrm{(b)} \mathrm{An} \mathrm{equivalent} \mathrm{circuit}$ used in the CNLS fitting procedure.

indicating that the kinetics of the oxidation process is limited by the diffusion of reactants inside the pores of the passive film. The low frequency capacitive $\left(Q_{4}\right)$ and Warburg $\left(Q_{5}\right)$ responses may be related to the intermediate species generated during the oxidation process and subsequently adsorbed on the electrode as well as diffusion-related components in outer pores, respectively. The reaction taking place at $0.24 \mathrm{~V}$ is most likely a mixed reaction of nickel hydroxide phase transformation from $\alpha$ to $\beta$ and nickel hydroxide oxidation.

Fig. 3 shows complex impedance spectra recorded at applied potentials between 0.32 and $0.42 \mathrm{~V}$. With increasing anodic polarization, nickel oxyhydroxide is generated from nickel hydroxide. Generally, CPE $\mathrm{Q}_{4}$ displays a pseudocapacitance response as the applied potential increases. The value of polarization resistance $\left(R_{P}=R_{1}\right)$ decreases as the

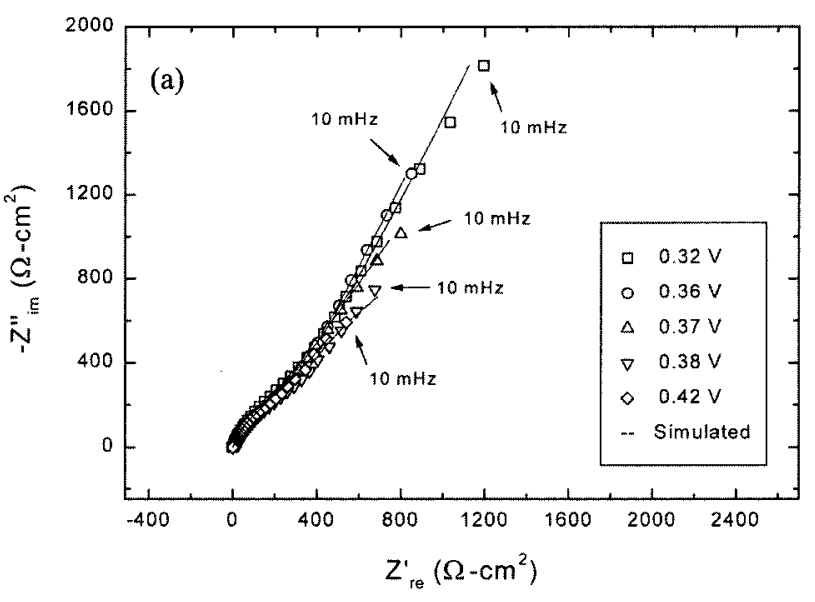

(b)

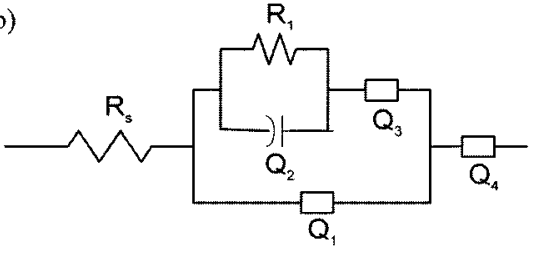

(c)

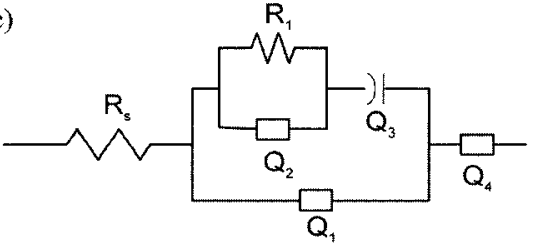

(d)

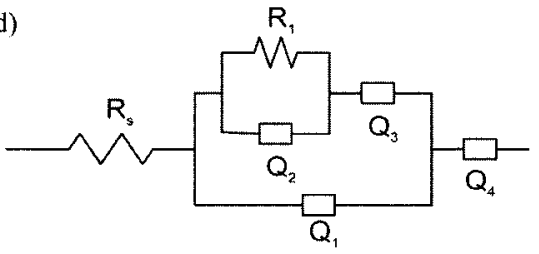

Fig. 3. (a) Complex impedance spectra for oxidation of nickel electrode between 0.32 and $0.42 \mathrm{~V}$ in $1 \mathrm{M} \mathrm{KOH.} \mathrm{(b)} \mathrm{An} \mathrm{equivalent}$ circuit for the data shown in (a).

potential increases to $0.37 \mathrm{~V}$. A reasonably small $R_{P}$ value of $660 \Omega$ is observed at $0.37 \mathrm{~V}$. From then on, it increases again, suggesting that the electrode is passivated (Table $\mathrm{I}$ ). 

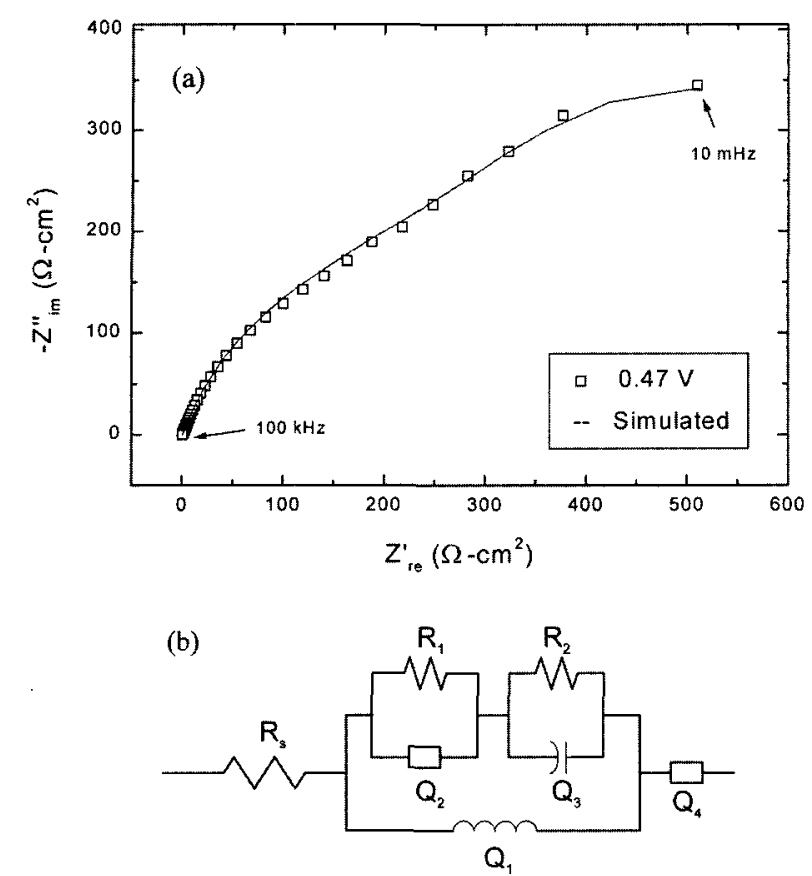

Fig. 4. (a) Complex impedance spectrum for oxidation of nickel electrode at $0.47 \mathrm{~V}$ in $1 \mathrm{M} \mathrm{KOH}$. (b) An equivalent circuit for the data shown in (a).

The complex impedance spectrum at $0.47 \mathrm{~V}$ is shown in Fig. 4. Two continuous semicircles are shown in the equivalent circuit. This indicates that two separate faradaic reactions may take place at this potential. The semicircle at high frequencies (the $R_{1}-Q_{2}$ parallel network in the equivalent circuit) is attributed to the oxidation while the second in the medium frequency region (the $\mathrm{R}_{2}-\mathrm{Q}_{3}$ parallel network) may be due to the oxygen evolution reaction. The $C P E Q_{3}$, which shows a Warburg-like behavior with $n$ values of 0.48 and 0.54 at 0.38 and $0.42 \mathrm{~V}$, respectively, changes to a double-layer capacitor with an $n$ value of 1.00 at $0.47 \mathrm{~V}$. From Table I, the inductor shown in the medium frequency range with an $n$ value of -1.00 suggests that the rapid transformation of nickel oxyhydroxide to higher valence oxide in the oxygen evolution region is impeded by the inductive current flowing in the opposite direction. The CPE $\mathrm{Q}_{2}$ is basically a double-layer capacitor with an $n$ value of 0.81 in the high frequency region and $\mathrm{Q}_{3}$ at medium frequencies shows either capacitive properties with large capacitance values or Warburg behaviors, depending on the potential applied. This suggests that the $\mathrm{NiOOH}$ film has large amounts of pores through which reactants diffuse in or a passive film acting as a capacitor, depending on the applied potential. The charge-transfer resistance of the second semicircle has a large value of $5817 \Omega$ suggesting that the oxygen evolution reaction requires a large overpotential.

Fig. 5 shows typical Bode plots of the phase angle vs. $\log$ $\omega$ in $1 \mathrm{M} \mathrm{KOH}$, where $\omega$ is the angular frequency $(2 \pi f)$ and the phase angle is the arc tangent of the ratio of the imaginary and real parts of the measured impedance. It is clear from this figure that the electrode reaction is made of more than one process in most cases.

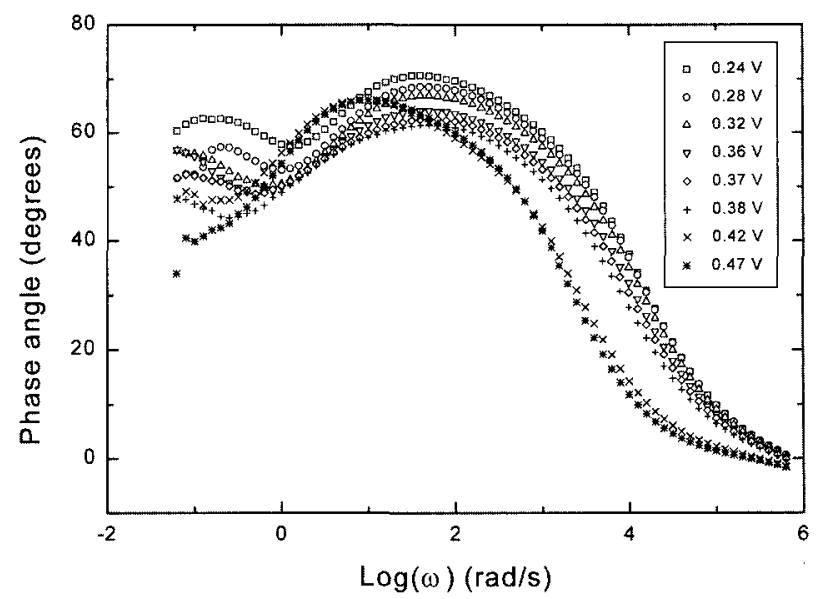

Fig. 5. Bode plot (phașe angle vs. $\log \omega$ ) at applied potentials in $1 \mathrm{M}$ KOH.

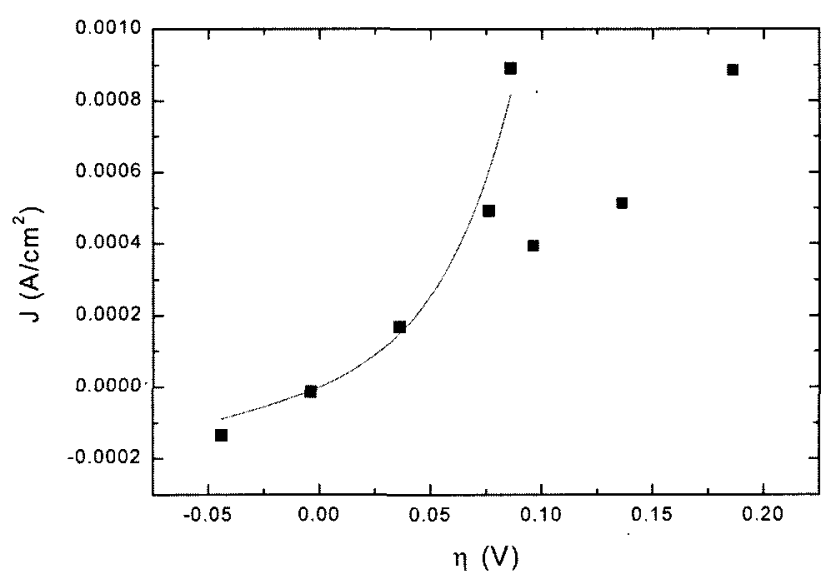

Fig. 6. The current density vs. overpotential plot in $1 \mathrm{M} \mathrm{KOH}$. The nonlinear least squares fitting curve has been drawn for the data obtained between 0.32 and $0.37 \mathrm{~V}$ with the Butler-Volmer equation.

A plot of current density (J) $v s$. overpotential ( $\eta$ ) was obtained using the data listed in Table $I$ and shown in Fig. 6. The formal potential $\left(\mathrm{E}^{0}\right)$ for oxidation of $\mathrm{Ni}$ to its hydroxide was estimated by taking an average of the anodic and cathodic peak potentials. The current density was obtained from the overpotential applied and the polarization resistances listed in Table I. There are two regions as shown in this plot. The first region at less positive potentials should be due to the oxidation of nickel hydroxide to nickel oxyhydroxide, whereas the more positive region is characterized as the passivation process taking place due to further oxidation. While the CV in Fig. 1 shows the sum of all currents including nonfaradaic components as well as those from other processes, the $J-\eta$ curve shown in Fig. 6 shows only the Faradaic current for oxidation of nickel. It is seen in Fig. 6 that the potential where the passivation starts is clearly shown.

The nonlinear least square regression between 0.24 and $0.37 \mathrm{~V}$ was used to evaluate kinetic parameters. The exchange current density, $i_{0}$, for the rate limiting one-electron chargetransfer reaction (see reaction 1 below) was calculated to be $7.0 \times 10^{-5} \mathrm{~A} / \mathrm{cm}^{2}$ with a transfer coefficient $(\alpha)$ value of 0.25 


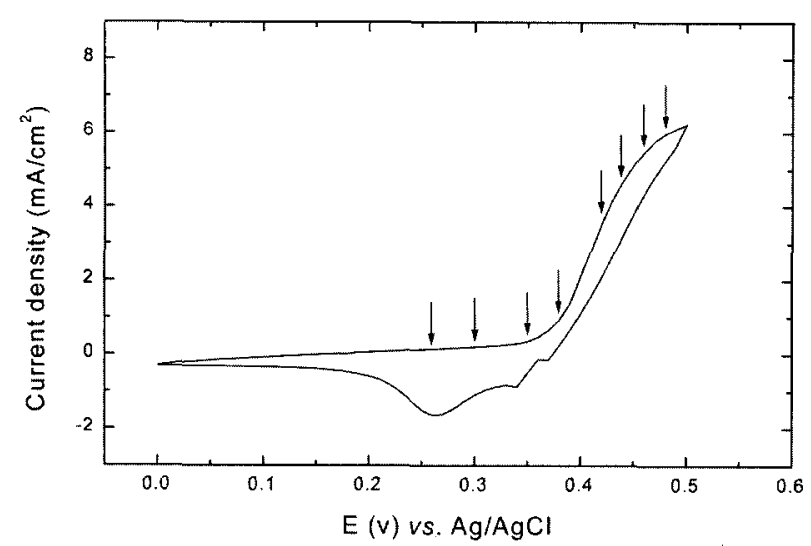

Fig. 7. Cyclic voltammogram of a nickel electrode in $1 \mathrm{M} \mathrm{KOH}$ solution containing $0.20 \mathrm{M}$ ethanol at a scan rate of $50 \mathrm{mV} / \mathrm{s}$. Impedance measurements were made at potentials marked. Electrode area was $0.15 \mathrm{~cm}^{2}$.

from the nonlinear fitting of the J- $\eta$ plot according to the Butler-Volmer equation. ${ }^{35}$ The irreversible shape of the CV shown in Fig. 1 supports that the $\alpha$-value should deviate significantly from an ideal case of 0.50 .

Impedance measurements for ethanol oxidation - A steadystate $\mathrm{CV}$ obtained at the nickel electrode in $1 \mathrm{M} \mathrm{KOH}$ solution containing $0.20 \mathrm{M}$ ethanol is shown in Fig. 7. The electrochemical impedance spectra for the oxidation of ethanol at the nickel electrode in $1 \mathrm{M} \mathrm{KOH}$ containing $0.20 \mathrm{M}$ ethanol were recorded between 0.26 and $0.48 \mathrm{~V} v s$. $\mathrm{Ag} / \mathrm{AgCl}$ at potentials marked on the $\mathrm{CV}$. All the parameters obtained from the simulation are listed in Table II. The equivalent circuits were derived from the simulation of the experimental data. The simulated data fit well to the experimental data.

The impedance spectrum recorded at $0.26 \mathrm{~V}$ is shown in Fig. 8. In the potential range measured, the $R_{S}$ value was $3.9 \pm 0.1 \Omega$, which is practically the same as that obtained without ethanol. The two R-Q networks at this potential are shown in Fig. $8 \mathrm{~b}$. The high frequency response is the same as those of nickel oxidation at 0.24 and $0.28 \mathrm{~V}$ in $1 \mathrm{M} \mathrm{KOH}$ (see Fig. 2b). The reaction taking place at $0.26 \mathrm{~V}$ is thus most

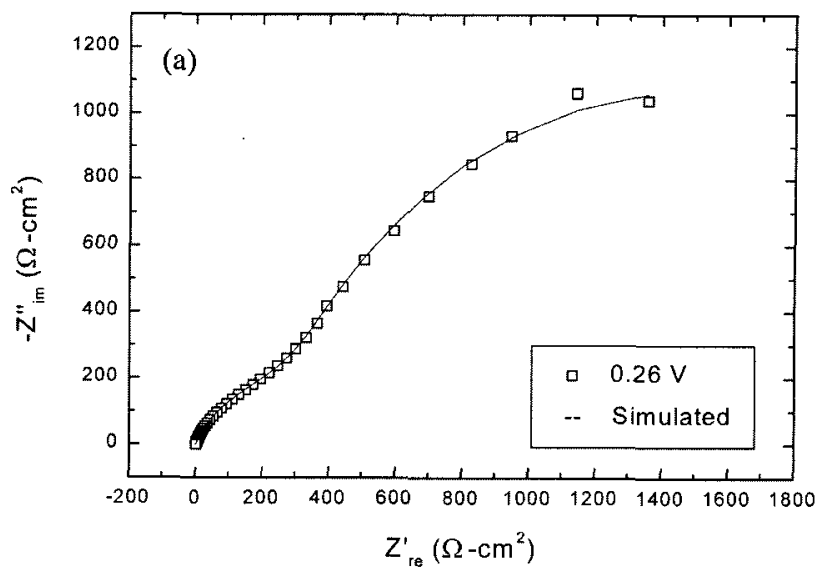

(b)

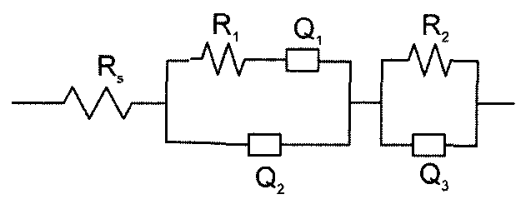

Fig. 8. (a) Complex impedance spectrum for oxidation of nickel electrode at $0.26 \mathrm{~V}$ in $1 \mathrm{M} \mathrm{KOH}$ containing $0.20 \mathrm{M}$ ethanol. (b) An equivalent circuit for the data shown in (a).

likely a mixed one of nickel hydroxide and ethanol oxidation, as the potential applied here is well beyond the thermodynamic potential for ethanol oxidation. The low frequency spectra may thus result from the adsorption of intermediates during nickel hydroxide oxidation and ethanol. These responses were observed up to $0.30 \mathrm{~V}$.

Fig. 9 shows the impedance spectra recorded between 0.38 and $0.44 \mathrm{~V}$. The impedance spectrum at $0.35 \mathrm{~V}$ is practically the same as the ones shown here except that the diameter of the depressed semicircle is larger. The polarization resistance, $R_{1}$, at high frequencies decreases sharply with an increasing overpotential. The low frequency capacitive response $\left(\mathrm{Q}_{2}\right)$ may be related to the adsorbed intermediate species generated during the oxidation of ethanol. Thus, the diffusion process may limit the oxidation reaction of ethanol due to this adsorp-

Table II. Parameters of Equivalent Circuits Obtained for Simulation of the Elements in $1 \mathrm{M} \mathrm{KOH} \mathrm{Containing} \mathrm{0.20} \mathrm{M} \mathrm{Ethanol.}$

\begin{tabular}{ccccccccccccc}
\hline & & \multicolumn{1}{c}{} & \multicolumn{3}{c}{$\mathrm{Q}_{1}$} & \multicolumn{2}{c}{$\mathrm{Q}_{2}$} & \multicolumn{3}{c}{$\mathrm{Q}_{3}$} \\
\hline $\mathrm{E}_{\text {applied }}(\mathrm{V})$ & $\mathrm{R}_{\mathrm{P}}(\Omega)^{\mathrm{b}}$ & $\mathrm{R}_{2}(\Omega)$ & $\mathrm{Y}_{\mathrm{Q}} \times 10^{-5}$ & $n$ & $\mathrm{Y}_{\mathrm{Q}} \times 10^{-5}$ & $n$ & $\mathrm{Y}_{\mathrm{Q}} \times 10^{-4}$ & $n$ & $\mathrm{Y}_{\mathrm{Q}} \times 10^{-4}$ & $n$ & $\tau(\mathrm{s})$ & $\chi^{2}\left(\times 10^{-4}\right)^{\mathrm{c}}$ \\
\hline 0.26 & $16259.0^{\mathrm{c}}$ & 2288.5 & 16259.0 & - & 117.62 & -0.90 & 2.63 & 0.74 & 9.14 & 0.91 & 0.521 & 1.74 \\
0.30 & $11548.0^{\mathrm{c}}$ & 1883.4 & 11548.0 & - & 3.01 & 0.74 & 8.68 & 0.88 & - & - & 0.554 & 2.14 \\
0.35 & 4527.0 & 1436.5 & 3090.5 & - & 5.32 & 0.70 & 15.43 & 0.87 & - & - & 0.441 & 5.17 \\
0.38 & 755.9 & 650.8 & 105.1 & - & 8.88 & 0.69 & 87.80 & 1.00 & - & - & 0.227 & 3.42 \\
0.42 & 298.8 & 12.8 & 286.0 & - & 26.92 & 0.76 & 10.65 & 0.80 & - & - & 0.113 & 2.38 \\
0.44 & 315.9 & 18.5 & 297.4 & - & 22.68 & 0.76 & 9.87 & 0.85 & - & - & 0.116 & 5.05 \\
0.46 & 517.5 & 102.8 & 414.7 & 22.0 & 21.08 & 0.67 & 10.76 & 0.93 & -892.38 & 0.32 & 0.229 & 2.05 \\
0.48 & 1431.5 & 548.9 & 882.6 & 130.2 & 4.90 & 0.57 & 9.36 & 0.82 & -198.7 & 00.43 & 0.604 & 3.70 \\
\hline
\end{tabular}

${ }^{a} R_{S}$ values are not listed as they are almost constant at $3.9 \pm 0.1 \Omega$ for measurements made between 0.26 and $0.48 \mathrm{~V}$.

${ }^{b} R_{P}$ is $R_{1}+R_{2}$ at all applied potentials.

${ }^{c} R_{P}$ here is $R_{2}$.

${ }^{d}$ Chi square values for the equivalent circuits fitting.

Electrode area was $0.15 \mathrm{~cm}^{2}$. 


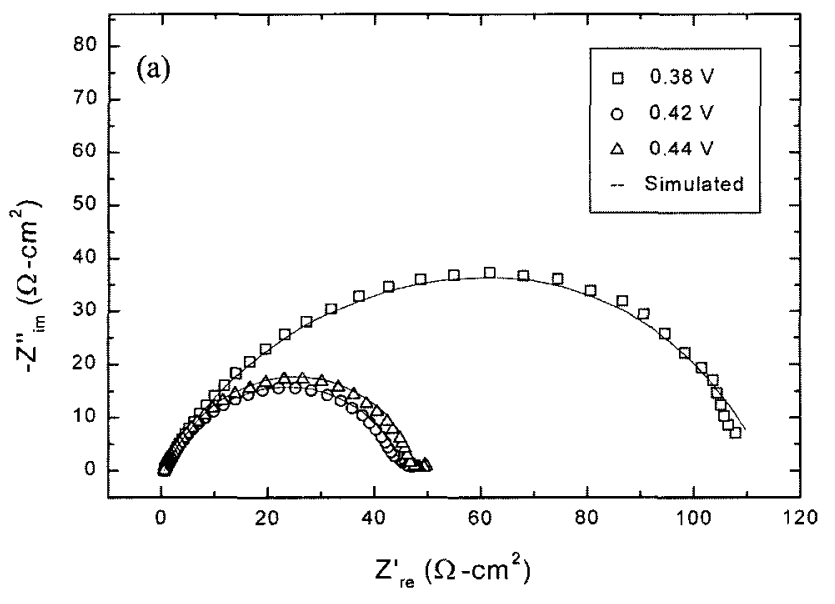

(b)

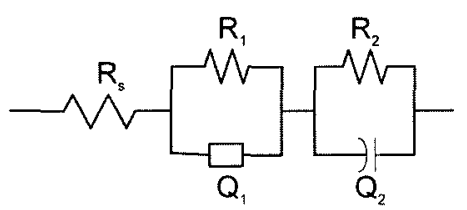

Fig. 9. (a) Complex impedance spectra for oxidation of nickel electrode at $0.38,0.42$, and $0.44 \mathrm{~V}$ in $1 \mathrm{M} \mathrm{KOH}$ containing $0.20 \mathrm{M}$ ethanol. (b) An equivalent circuit for the data shown in (a).

tion, and the CPE has an intermediate character $(n=0.85)$ between a capacitor $(n=1)$ and a Warburg response $(n=0.5)$. The $R_{p}$ values increase above $0.42 \mathrm{~V}$, which indicates that oxidation of ethanol above this potential is impeded by the passivation of $\mathrm{NiOOH}$. $\beta-\mathrm{NiOH}$ is oxidized to higher oxidation states (i.e., Ni(IV)) in the oxygen evolution region. ${ }^{8}$ This causes a considerable increase in the film thickness and a remarkable drop in the electronic conductivity of the film, as was shown by spectroelectrochemical ${ }^{8}$ and ellipsometric experiments. ${ }^{36}$ Also, the $\mathrm{Ni}(\mathrm{OH})_{2}$ regenerated during ethanol oxidation would be accumulated on the electrode surface as the potential increases. Therefore, the polarization resistance would also be increased due to the decreasing electronic conductivity caused by $\mathrm{Ni}(\mathrm{OH})_{2}$ at higher potential. ${ }^{37,41}$

The impedance spectra composed of three $\mathrm{RC}$ networks observed at 0.46 and $0.48 \mathrm{~V}$ are shown in Fig. 10. Oxidation of nickel oxyhydroxide observed as was shown in Fig. 4 is not observed here. This suggests that the redox couple of $\beta$ $\mathrm{Ni}(\mathrm{OH})_{2} / \beta-\mathrm{NiOOH}$ is acting as an effective electron transfer mediator for ethanol oxidation. In this region, electrogenerated $\beta-\mathrm{NiOOH}$ is reduced to $\beta-\mathrm{Ni}(\mathrm{OH})_{2}$ by ethanol oxidation. The third $\mathrm{RC}$ network may represent the oxygen evolution process. The $\mathrm{CPE} \mathrm{Q}_{3}$ is negative and shows a Warburg-like behavior with $n$ values of 0.32 and 0.43 .

Bode plots of the phase angles vs. $\log \omega$ generally display two maxima between 0.26 and $0.35 \mathrm{~V}$ (not shown), indicating that there are two independent processes going on in this potential region. In the potential region where ethanol oxidation is efficient, the two processes merge into one, suggesting that the oxidation of ethanol by the electrogenerated $\mathrm{NiOOH}$ is very fast.

A plot of the current density vs. overpotential obtained

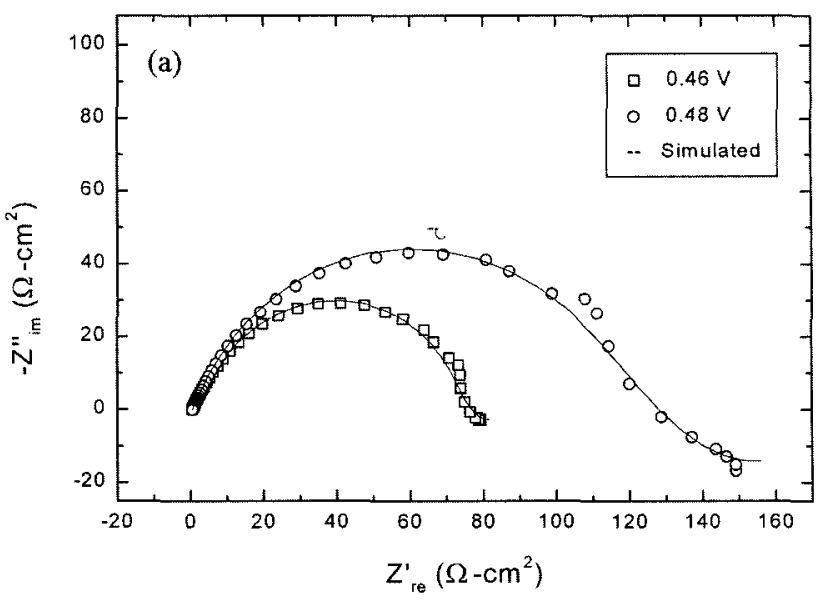

(b)

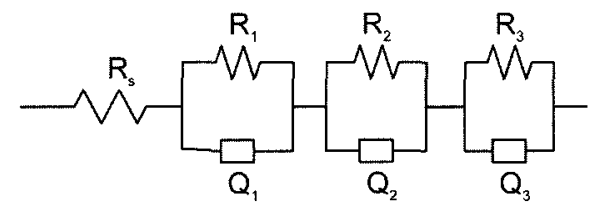

Fig. 10. (a) Complex impedance spectra for oxidation of nickel electrode at 0.46 and $0.48 \mathrm{~V}$ in $1 \mathrm{M} \mathrm{KOH}$ containing $0.20 \mathrm{M}$ ethanol. (b) An equivalent circuit for the data shown in (a).

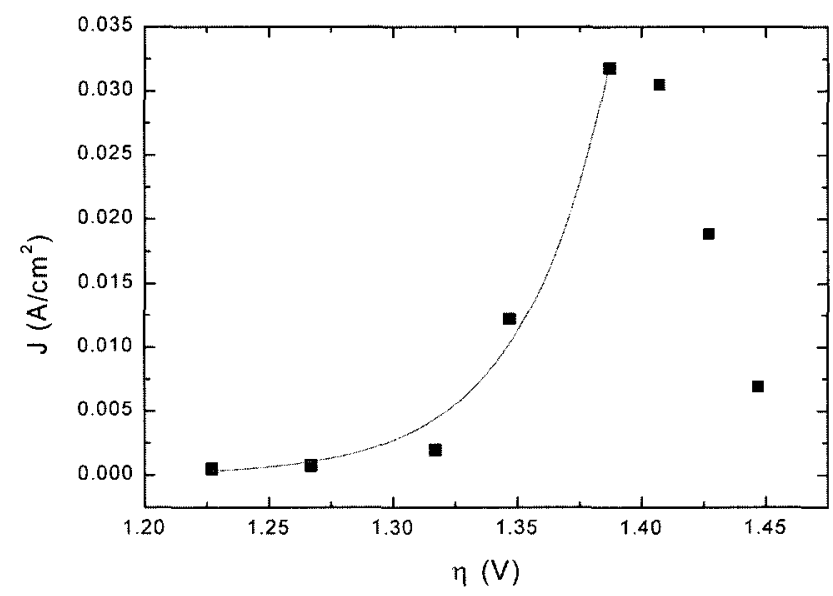

Fig. 11. The current density vs. overpotential plot in $1 \mathrm{M} \mathrm{KOH}$ containing $0.20 \mathrm{M}$ ethanol. The nonlinear least squares fitting curve has been drawn for the data obtained between 0.35 and $0.42 \mathrm{~V}$ using the Butler-Volmer equation.

using the data listed in Table II is shown in Fig. 11. In the region of low anodic potentials, ethanol oxidation follows the Butler-Volmer relation, whereas the electrode is shown to be passivated beyond about $\eta \cong 1.37 \mathrm{~V}$. A nonlinear least square regression between 0.35 and $0.42 \mathrm{~V}$, which represents the data before passivation began, was used to evaluate the kinetic parameters of ethanol oxidation. The exchange current density, $i_{0}$, obtained from the nonlinear least square regression for oxidation of ethanol) is determined to be $2.7 \times 10^{-19} \mathrm{~A} / \mathrm{cm}^{2}$ at the equilibrium potential, which is estimated to be $-0.967 \mathrm{~V} v s$. $\mathrm{Ag} / \mathrm{AgCl}$ at the $\mathrm{pH}$ used in this work, ${ }^{38-40}$ and the transfer coefficient, $\alpha$, is 0.27 . 


\section{Discussion}

Oxidation of nickel hydroxide $-\alpha-\mathrm{Ni}(\mathrm{OH})_{2}$ generated from nickel oxidation is known to slowly transform to $\beta-\mathrm{Ni}(\mathrm{OH})_{2}$. The $\beta-\mathrm{Ni}(\mathrm{OH})_{2}$ is oxidized to $\beta$-phase oxyhydroxide at a relatively low anodic overpotential. ${ }^{2-8}$ In the potential region of oxygen evolution, $\beta-\mathrm{NiOOH}$ is further oxidized to nickel(IV) oxide and other intermediate species on the surface. In the oxidation reaction of $\beta-\mathrm{Ni}(\mathrm{OH})_{2}$ to $\beta-\mathrm{NiOOH}$, proton insertion and de-insertion in the solid phase is the rate-determining step. ${ }^{41}$ The proton diffusion coefficient is an important factor in this process. ${ }^{3,7,42}$ The proton diffusion coefficient, D, has been reported to be $10^{-9}$ and $10^{-11} \mathrm{~cm}^{2} / \mathrm{s}$ for the reversible $\mathrm{e}^{3}$ and irreversible ${ }^{7}$ reactions, respectively, from CV studies and 4.6 $\times 10^{-11}$ and $1.0 \times 10^{-12} \mathrm{~cm}^{2} / \mathrm{s}$, respectively, assuming reversible kinetics from chronoamperometry studies. Ta and Newman ${ }^{42}$ reported proton diffusion coefficients ranging $1.2 \times 10^{-13}$ to $1.9 \times 10^{-12} \mathrm{~cm}^{2} / \mathrm{s}$ depending on the proton concentration in nickel hydroxide thin films with their thickness ranging between $10 \sim 40 \mathrm{~nm}$. Motupally et al. reported that D is between $10^{-8}$ and $10^{-11} \mathrm{~cm}^{2} / \mathrm{s}$ as a function of the state of charge from the impedance measurements. ${ }^{43}$ The $\mathrm{D}$ values vary with the potential of film formation and with the oxidation state of the nickel. We also estimated the diffusion coefficient from the equation, $\mathrm{D}=l^{2} \cdot \omega / 5.12$, proposed by Armstrong ${ }^{44}$ and discussed by Mathias and Haas, ${ }^{45}$ where $\omega$ is the angular frequency in the transition region of the complex impedance spectrum with a slope of -2.0 and $l$ is the film thickness. The $l$-values of $13 \mathrm{~nm}^{7}$ and $40 \mathrm{~nm}^{42}$ yielded the proton diffusion coefficients of $(4.7 \pm 0.6) \times 10^{-14}$ and $(4.4 \pm 0.6) \times 10^{-13} \mathrm{~cm}^{2} / \mathrm{s}$, respectively, at the $\omega$-value of 0.125 and $0.158 / \mathrm{s}$ when the potential was stepped from 0.32 to $0.37 \mathrm{~V}$. The large variations in diffusion coefficients of as large as five orders of magnitudes could have resulted from parameters taken into account during the measurements or estimations, including: various assumptions made; in-accuracies of experimental parameters such as $l$; variations in phases $(\alpha-, \beta-$, or mixed) depending on the aging period after the hydroxide or oxides have been prepared; and different potential gradients between the two walls of the oxide/ hydroxide layer. The potential gradient should also affect the diffusion coefficient of the proton because of its high charge density. Therefore, the electron-transfer for reaction [1] must be fast but limited by proton diffusion; the Warburg response $\left(Q_{3}\right)$ observed in Fig. $3 \mathrm{~b}$ and $\mathrm{d}$ supports this.

Simple equivalent $\mathrm{RC}$ circuits were used in the simulation of most of the electrochemical interfaces for nickel oxidation in earlier studies. ${ }^{15,16.19,23}$ However, these circuits are not suitable for explaining the complex nickel oxidation process. We constructed expanded equivalent circuits in our current work to simulate our experimental data, which explain our experimental data rather well. These expanded equivalent circuits consist of one RC network and appropriate combination of various other components.

Oxidation of ethanol at oxide films - The ethanol oxidation mechanism at the $\mathrm{Ni}$ electrode in alkaline media has been proposed by Fleishmann et al.' The proposed mechanism may be summarized as following:

$$
\begin{aligned}
& \mathrm{Ni}(\mathrm{OH})_{2}+\mathrm{OH}^{-} \Leftrightarrow \mathrm{NiOOH}+\mathrm{H}_{2} \mathrm{O}+\mathrm{e}^{-} \\
& \mathrm{NiOOH}+\mathrm{CH}_{3} \mathrm{CH}_{2} \mathrm{OH} \rightarrow \text { Intermediate } 1+\mathrm{Ni}(\mathrm{OH})_{2} \\
& \mathrm{NiOOH}+\text { Intermediate } 1 \rightarrow \mathrm{CH}_{3} \mathrm{CHO}+\mathrm{Ni}(\mathrm{OH})_{2} \\
& \mathrm{NiOOH}+\mathrm{CH}_{3} \mathrm{CHO} \rightarrow \text { Intermediate } 2+\mathrm{Ni}(\mathrm{OH})_{2} \\
& \mathrm{NiOOH}+\text { Intermediate } 2 \rightarrow \mathrm{CH}_{3} \mathrm{COOH}+\mathrm{Ni}(\mathrm{OH})_{2}
\end{aligned}
$$

The conversion of ethanol to acetic acid was reported to be $98 \%{ }^{1,46}$ Therefore, we also assumed that ethanol is completely oxidized to acetic acid at the nickel electrode. The exchange current density is very small at $2.7 \times 10^{-19} \mathrm{~A} / \mathrm{cm}^{2}$. This is because the oxidation potential of the nickel hydroxide/ oxyhydroxide redox couple is too far away from that of thermodynamic potential of ethanol oxidation, $-0.967 \mathrm{~V}$ vs. the $\mathrm{Ag} / \mathrm{AgCl}$ electrode. The ethanol is not oxidized until nickel hydroxide undergoes oxidation. For this reason, the $\alpha$-value for the oxidation of ethanol is practically the same as that for nickel oxidation. In other words, the activation barrier for the alcohol oxidation is essentially the same as that for nickel oxidation. The only kinetic parameter that is different between these two oxidation systems is the exchange current, indicating that alcohol is oxidized only after nickel hydroxide is oxidized. This also indicates that the rate limiting step for alcohol oxidation at the nickel electrode is the oxidation of nickel hydroxide to a higher valance oxides/oxyhydroxide. Therefore, the nickel electrode is regarded as a good electrocatalyst for the electrooxidation of ethanol.

\section{Conclusion}

The electrochemical oxidation of nickel hydroxide in $1 \mathrm{M}$ $\mathrm{KOH}$ was first studied between 0.24 and $0.47 \mathrm{~V}$ vs. $\mathrm{Ag} / \mathrm{AgCl}$ by electrochemical impedance spectroscopy. The maximum rate of oxidation takes place at $0.37 \mathrm{~V}$, represented by the minimum $R_{P}$ value and the passivation of the electrode starts at a more positive potential. Nickel hydroxide/oxyhydroxide/ oxide transitions were observed at more positive potentials. The exchange current for the nickel hydroxide/nickel oxyhydroxide transition was estimated to be $7.0^{\circ} ø 10^{-5} \mathrm{~A} / \mathrm{cm}^{2}$ with an $\alpha$ value of 0.25 .

The electrochemical oxidation of ethanol in $1 \mathrm{M} \mathrm{KOH}$ containing $0.20 \mathrm{M}$ ethanol was studied next between 0.26 and $0.48 \mathrm{~V}$ vs. $\mathrm{Ag} / \mathrm{AgCl}$. The sizable oxidation current for ethanol occurred at a potential more positive than $0.35 \mathrm{~V}$. The maximum rate of oxidation of ethanol was observed at $0.42 \mathrm{~V}$, represented by the minimum $R_{P}$ value and the oxidation of ethanol is limited by the diffusion of adsorbed intermediates. Our results indicate that the rate limiting step for alcohol oxidation is the oxidation of nickel itself. This result suggests that finding a metal electrode undergoing oxidation at less anodic potentials and yet capable of mediating organic compounds is very important in reducing the overpotential for oxidation of organic compounds, when efficient mediated oxidation of organic fuels is to be obtained for a more efficient fuel cell design. 


\section{Acknowledgment}

This work was supported by a grant from KOSEF through the Center for Integrated Molecular Systems at the Pohang University of Science and Technology and by the BK21 program of the Korea Research Foundation. The graduate stipends were supported by the BK-21 program of KRF.

\section{References}

1. M. Fleischmann, K. Korinek, and D. Pletcher, J. Chem. Soc. Perkin II, 1396 (1972).

2. H. Bode, K. Delhmelt, and J. Whitte, "Zur kenntnis der nickelhydroxidelektrode?I. Uber das nickel (II)-hydroxidhydrat", Electrochim. Acta, 11, 1079 (1966).

3. D. M. MacArthur, J. Electrochem. Soc., 117, 422 (1970).

4. R. S. Schrebler Guzman, J. R. Vilche, and A. J. Arvia, J. Electrochem. Soc., 125, 1578 (1978).

5. R. Barnard and C. F. Randell, J. Appl. Electrochem., 13, 89 (1983).

6. R. Barnard and C. F. Randell, J. Appl. Electrochem., 13, 97 (1983).

7. C. Zhang and S.-M. Park, "The anodic oxidation of nickel in alkaline media studied by spectroelectrochemical techniques", $J$. Electrochem. Soc., 134, 2966 (1987).

8. C. Zhang and S.-M. Park, "In-situ spectroelectrochemical studies on the anodic oxidation of nickel hydroxide in alkaline media", $J$. Electrochem. Soc., 136, 3333 (1989).

9. J. McBreen, W. E. O'Grady, G Tourillon, E. Dartyge, A. Fontaine, and K. I. Pandya, "In situ time-resolved x-ray absorption near edge structure study of the nickel oxide electrode", J. Phys. Chem., 93, 6308 (1989).

10. K. I. Pandya, W. E. O'Grady, D. A. Corrigan, J. McBreen, and R. W. Hoffman, "Extended x-ray absorption fine structure investigations of nickel hydroxides", J. Phys. Chem., 94, 21 (1990).

11. K. I. Pandya, R. W. Hoffman, J. McBreen, and W. E. O'Grady, J. Electrochem. Soc., 137, 383 (1990).

12. D. Guay, G. Tourillon, E. Dartyge, A. Fontaine, J. McBreen, K. I. Pandya, and W. E. O'Grady, "In-situ time-resolved EXAFS study of the structural modifications occurring in nickel oxide electrodes between their fully oxidized and reduced states", J. Electroanal. Chem., 305, 83 (1991).

13. X. Qian, H. Sambe, D. E. Ramaker, K. I. Pandya, and W. E. O'Grady, J. Phys. Chem., 101, 9441 (1997).

14. S. H. Glarum and J. H. Marshall, J. Electrochem. Soc., 129, 535 (1982).

15. S. J. Lenhart, D. D. Macdonald, and B. G Pound, J. Electrochem. Soc., 135, 1063 (1988).

16. A. Gorenstein, F. Decker, W. Estrada, C. Esteves, A. Andersson, S. Passerini, S. Pantaloni, and B. Scrosati, "Electrochromic NiOxHy, hydrated films: cyclic voltammetry and ac impedance spectroscopy in aqueous electrolyte" , J. Electroanal. Chem., 277, 277 (1990).

17. S. D. Bhakta, D. D. Macdonald, B. G. Pound, and M. UrquidiMacdonald, J. Electrochem. Soc., 138, 1353 (1991).

18. D. D. Macdonald, M. Urquidi-Macdonald, S. D. Bhakta, and B. G Pound, J. Electrochem. Soc., 138, 1359 (1991).

19. M. A. Reid and P. L. Loyselle, "Impedances of nickel electrodes cycled in various KOH concentrations" , J. Power Sources, 36, 285 (1991).

20. M. S. Suresh, "Double-layer impedance of a nickel oxide electrode at low states-of-charge" , J. Power Sources, 47, 27 (1994).

21. M. Cappadonia, J. Divisek, T. von der Heyden, and U. Stimming, "Oxygen evolution at nickel anodes in concentrated alkaline solution", Electrochim. Acta, 39, 1559 (1994).

22. G. Barral, F. Njanjo-Eyoke, and S. Maximovitch, "Characterisation of the passive layer and of hydroxide deposits of nickel by impedance spectroscopy", Electrochim. Acta, 40, 2815 (1995).

23. V. Mancier, A. Metrot, and P. Willmann, "Ac impedance modelling of nickel hydroxide electrodes viewed as mixed protonic-electronic conductors", Electrochim. Acta, 41, 1259 (1996).

24. G. Barral, S. Maximovitch, and F. Njanjo-Eyoke, "Study of electrochemically formed $\mathrm{Ni}(\mathrm{OH}) 2$ layers by EIS", Electrochim. Acta, 41, 1305 (1996).

25. S. Maximovitch, "Influence of formation conditions on impedance properties of nickel passive layers formed in $1 \mathrm{M} \mathrm{KOH",} \mathrm{Electro-}$ chim. Acta, 41, 2761 (1996).

26. C. Y. Chao, L. F. Lin, and D. D. Macdonald, J. Electrochem. Soc., 128, 1187 (1981).

27. L. F. Lin, C. Y. Chao, and D. D. Macdonald, J. Electrochem. Soc., 128, 1194 (1981).

28. C. Y. Chao, L. F. Lin, and D. D. Macdonald, J. Electrochem. Soc, 129, 1874 (1982).

29. J.-W. Kim and S.-M. Park, "Electrochemical Oxidation of Ethanol at Thermally Prepared $\mathrm{RuO}_{2}$-Modified Electrodes in Alkaline Media", J. Electrochem. Soc., 146, 1075 (1999).

30. B. A. Boukamp, Equivalent Circuit User's Manual, 2nd ed., University of Twente, Enschede, the Netherlands (1989).

31. B. J. Johnson and S.-M. Park, "Electrochemistry of Conductive Polymer XIX. Oxidation of Aniline at Bare and PolyanilineModified Platinum Electrodes Studied by Electrochemical Impedance Spectroscopy”, J. Electrochem. Soc., 143, 1269 (1996).

32. M. Cai and S.-M. Park, "Spectroelectrochemical studies on dissolution and passivation of zinc electrodes in alkaline solutions", J. Electrochem. Soc., 143, 2125 (1996).

33. D. K. Cha and S.-M. Park, "Electrochemical Oxidation of $\mathrm{Mn}(\mathrm{OH}) 2$ in Alkaline Media" , J. Electrochem. Soc., 144, 2573 (1997)

34. S.-M. Park and J.-S. Yoo, "Electrochemical impedance spectroscopy for better electrochemical measurements", Anal. Chem. 75, 455A (2003).

35. A. J. Bard and L. R. Faulkner, Electrochemical Methods, John Wiley \& Sons, Inc. (1980).

36. P. W. T. Lu and S. Srinivasan, J. Electrochem. Soc., 125, 1416 (1978).

37. Y. L. Lo and B. J. Hwang, J. Electrochem. Soc., 142, 445 (1995).

38. L. W. H. Leung, S. C. Chang, and M. J. Weaver, J. Electroanal. Chem., 266, 317 (1989).

39. P. Gao, S. C. Chang, Z. Zhou, and M. J. Weaver, "Electrooxidation pathways of simple alcohols at platinum in pure nonaqueous and concentrated aqueous environments as studied by real-time ftir spectroscopy" , J. Electroanal. Chem., 272, 161 (1989).

40. S.-M. Park, N. C. Chen, and N. Doddapaneni, "Electrochemical oxidation of ethanol in aqueous carbonate solutions", $J$. Electrochem. Soc., 142, 40 (1995).

41. J.-W. Kim and S.-M. Park, "In-situ XANES studies of electrodeposited nickel oxide films with metal additives for the electro-oxidation of ethanol", J. Electrochem. Soc., 150, E560 (2003).

42. K. P. Ta and J. Newman, "Mass Transfer and Kinetic Phenomena at the Nickel Hydroxide Electrode", J. Electrochem. Soc., 145, 3860 (1998).

43. S. Motupally, C. C. Streinz, and J. W. Weidner, J. Electrochem. Soc., 142, 1401 (1995).

44. R. D. Armstrong, "Impedance plane display for an electrode with diffusion restricted to a thin layer" , J. Electroanal. Chem., 198, 177 (1986).

45. M. F. Mathias and O. Haas, "An alternating current impedance model including migration and redox-site interactions at polymermodified electrodes", J. Phys. Chem., 96, 3174 (1992).

46. G. Vertes and G. Horanyi, "Some problems of the kinetics of the oxidation of organic compounds at oxide-covered nickel electrodes", J. Electroanal. Chem., 52, 47 (1974). 